\title{
A Case of Acute Posterior Multifocal Placoid Pigment Epitheliopathy Demonstrating Vogt-Koyanagi- Harada Disease-Like Optical Coherence Tomography Findings in the Acute Stage
}

\author{
Minoru Tanigawa Yoko Tsukahara Hiromitsu Yamanaka \\ Department of Ophthalmology, Shin-Nagata Eye Institute, Kobe, Japan
}

\section{Key Words}

Acute posterior multifocal placoid pigment epitheliopathy · Pigment epitheliopathy - Optical coherence tomography · Vogt-Koyanagi-Harada disease

\begin{abstract}
Purpose: We report a case of acute posterior multifocal placoid pigment epitheliopathy (APMPPE), which was difficult to differentiate from posterior pole-type Vogt-KoyanagiHarada (VKH) disease because the lesions were mainly located in the macula bilaterally. Case Report: A 33-year-old man presented with rapid bilateral loss of vision. Fundoscopy revealed yellow-white subretinal lesions in the posterior pole of both eyes. Optical coherence tomography (OCT) revealed the presence of subretinal fluid with a subretinal septum. After initiation of systemic steroids, OCT revealed that the amount of subretinal fluid decreased immediately. However, vision loss was less responsive to the therapy, and OCT revealed partial reorganization of the inner segment/outer segment (IS/OS) line in the bilateral macular areas after therapy. Discussion: In our case, the location of the macular lesions made it difficult to differentiate APMPPE from VKH disease by fluorescein angiography. OCT images showed VKH disease-like findings of serous retinal detachment with a subretinal septum. The outer nuclear layer disappeared and the IS/OS line in the affected area was disorganized in the acute stage of the disease. In this case, the rapid loss of vision was specific to the onset pattern of APMPPE, and the slow response to therapy was very different
\end{abstract}


Tanigawa et al:: A Case of Acute Posterior Multifocal Placoid Pigment Epitheliopathy Demonstrating Vogt-Koyanagi-Harada Disease-Like Optical Coherence Tomography Findings in the Acute Stage

from the response typically observed in VKH disease. Thus, careful consideration of the clinical course is important for diagnosing APMPPE.

(c) 2013 S. Karger AG, Basel

\section{Introduction}

Acute posterior multifocal placoid pigment epitheliopathy (APMPPE) is a self-limiting chorioretinal inflammatory disease that was first described by Gass in 1968 [1]. APMPPE typically affects healthy young adults and is characterized by rapid loss of central vision and multiple inflammatory yellow-white lesions deep within the sensory retina at the level of the retinal pigment epithelium as well as classic fluorescein angiography (FA) findings. The subretinal lesions block most of the background choroidal fluorescence in the early phases of FA. Mid- and late-phase angiograms demonstrate diffuse and even staining of the acute lesions.

\section{Case Report}

A 33-year-old man presented with loss of vision in his right eye, which had developed the day before. Best-corrected visual acuity (BCVA) measured using the Landolt ring chart was 0.03 and 1.5 in the right and left eye, respectively (fig. 1, day $-3 ; 3$ days prior to steroid pulse therapy). No remarkable findings or inflammation were observed in the anterior segment of either eye. Fundoscopy revealed a yellow-white inflammatory subretinal lesion in the posterior pole of the right eye. Spectral-domain optical coherence tomography (SDOCT) showed the presence of subretinal fluid with a subretinal septum. No fundoscopy or SD-OCT was performed in the left eye at the first hospital visit. The next day, he presented with rapid loss of vision in his left eye, and BCVA in his left eye had decreased from 1.5 to 0.1 (fig. 1, day -2). Fundoscopy revealed a yellow-white lesion in the posterior pole of his left eye, which was similar to the lesion in his right eye (fig. 2a, b). SD-OCT images of both eyes showed serous retinal detachment with a subretinal septum and an intraretinal compartmentalized cystic space (fig. 3a, b). In his left eye, pigment epithelial detachment (PED) was observed on OCT (fig. 3b). The lesions in both eyes were limited to the macular area. Blood examination showed no remarkable abnormalities. Antibodies for Toxoplasma gondii were negative. Furthermore, the rapid plasma reagin test and fluorescent treponemal antibody absorption test were negative. The patient did not complain of abnormal skin sensations, headaches, hearing problems, or prodromal symptoms suggestive of viral infection. Apparent hypofluorescence was unremarkable in the early phase of FA (fig. 2c, d). Fluorescein dye in both eyes became gradually enhanced in the late phase of FA (not shown).

Steroid pulse therapy comprising methylprednisolone $(1,000 \mathrm{mg} /$ day) was intravenously administered for 3 days, followed by oral prednisolone for 17 weeks (tapering from 50 $\mathrm{mg}$ /day for 4 days, $40 \mathrm{mg} /$ day for 4 days, $30 \mathrm{mg} /$ day for 4 days, $20 \mathrm{mg} /$ day for 20 days, 15 $\mathrm{mg} /$ day for 28 days, $10 \mathrm{mg} /$ day for 48 days, and $5 \mathrm{mg} /$ day for 14 days). After initiation of systemic steroid therapy, SD-OCT revealed that the amount of subretinal fluid decreased immediately, but the inner segment/outer segment (IS/OS) line remained disorganized (fig. $3 c-f)$. Recovery of visual acuity was delayed compared to the rapid decrease in subretinal fluid. Goldmann perimetry performed 1 week after initiation of systemic steroid therapy revealed that a central relative scotoma persisted in both eyes (data not shown). Fifteen days after initiation of therapy, BCVA was 0.09 in both eyes (fig. 1). In addition, OCT showed that the IS/OS line remained disorganized, the retina appeared to be thin, and the outer 
Tanigawa et al:: A Case of Acute Posterior Multifocal Placoid Pigment Epitheliopathy Demonstrating Vogt-Koyanagi-Harada Disease-Like Optical Coherence Tomography Findings in the Acute Stage

nuclear layer seemed to be poorly organized (fig. 4a, b). Until day 33 of therapy, BCVA was $<0.1$ in both eyes, and it changed to 0.1 in both eyes on day 34 after therapy initiation (fig. 1). The response to therapy was slow and very different from that typically observed in VKH disease. We made the diagnosis of APMPPE on the basis of the rapid loss of vision bilaterally and the classic manifestation of yellow-white lesions in the fundus [1]. Eventually, vision began to recover and BCVA improved to 0.4 in the right eye and 0.5 in the left eye (fig. 1, day 48). At this time point, OCT revealed the partial recovery of the IS/OS line in the macular area of both eyes (fig. 4c, d). On day 111 of therapy initiation, BCVA improved to 0.7 in the right eye and 0.6 in the left eye (fig. 1), and OCT revealed clear reorganization of the IS/OS line (fig. 4e, f). One year after therapy initiation, BCVA remained 0.7 in the right eye and 1.0 in the left eye. However, unfortunately, we were unable to perform the OCT and examine the fundus.

\section{Discussion}

APMPPE can sometimes be difficult to differentiate from inflammatory diseases accompanied by retinal edema, such as VKH disease and ocular toxoplasmosis [2, 3]. The characteristic feature of the early phase of APMPPE hypofluorescence is considered secondary to either blocking of background choroidal fluorescence or choroidal hypoperfusion [4].

Initially, our patient was treated with systemic steroids for suspected posterior poletype VKH disease, which was diagnosed based on retinal edema on fundoscopy, serous retinal detachment with subretinal septum on OCT, and progressive pooling of dye on FA. The patient's response to therapy was slow: BCVA persisted at less than 0.1, and OCT revealed that the IS/OS line remained disorganized for 5 weeks after therapy initiation. It eventually began to reorganize 48 days after therapy initiation.

The conventional diagnostic method of reviewing the clinical course of rapid loss of vision in both eyes and the classic manifestation of a fundus lesion, described by Gass in 1968 [1], enabled us to make the right diagnosis. In this case, the rapid loss of vision was specific to the onset pattern of APMPPE, and the slow response to therapy was very different from the response typically observed in VKH disease. Our patient did not complain of headaches, hearing problems, or abnormal skin sensations, although these symptoms do not always accompany VKH disease.

We had difficulty detecting APMPPE-specific hypofluorescence in the early phase of FA because of the macular lesions, which showed a dark appearance due to the properties of the retinal pigment epithelium and the presence of xanthophyll pigment. In our case, the location of the macular lesions was one of the main reasons that made it difficult to differentiate APMPPE from VKH disease.

The use of steroid therapy for APMPPE remains controversial. Moreover, APMPPE has been known to resolve spontaneously. However, APMPPE is generally believed to be an inflammatory condition in which the patients suffer from rapid loss of central vision. Therefore, we believe that steroid therapy would be useful to shorten the duration of visual recovery in some cases. Furthermore, there has been no evidence of steroid therapy deteriorating the visual prognosis of patients with APMPPE.

The visual prognosis in APMPPE is strongly affected by the presence of initial foveal involvement. Previous studies have reported full visual recovery in $88 \%$ of eyes without initial foveal involvement and in 53\% of eyes with initial foveal involvement [5]. In our case, the patient's visual recovery was prolonged probably because of the initial foveal involvement despite of high-dose steroid therapy. 
Furthermore, OCT revealed serous retinal detachment with a subretinal septum typical of VKH disease. The aberrancies in the outer nuclear layer and the IS/OS line near the affected area were detected in the acute stage. Detailed observation of the SD-OCT images in the acute stage showed a retinal fluid space below the neurosensory retina that was divided by a septum, which seemed to be continuous with the IS/OS line, forming an intraretinal compartmentalized cystic space. Interestingly, in some slices of the SD-OCT images the compartmentalized intraretinal space contained two hyperreflective membranous layers during the acute stage. It remains unclear which layers of the normal retina correspond to these two membranous layers. These layers were disrupted into debris at later days, and the IS/OS line disappeared in the affected area. Our patient also showed foveal retinal detachment, which was first reported by Birnbaum et al. [3] who described the first series of APMPPE cases with foveal detachment. Thus far, OCT findings of APMPPE have shown that the outer nuclear layer, external limiting membrane, and the IS/OS line are disrupted $[2,3$, 6-10] and that serous retinal detachment occurs with or without intraretinal cystic space formation. Although hyperreflective debris or deposits were reportedly observed in the subretinal fluid or intraretinal cystic space $[2,3,8]$, to our knowledge, our case is the first to report disruption of the membranous layers in the intraretinal cystic space. Furthermore, in our patient, OCT of the left eye showed PED before therapy initiation. Lim et al. [10] reported that marked anterior displacement along with the neural retina was observed by OCT, and they considered that this was compatible with the enlargement of the underlying choroid by an inflammatory infiltrate associated with choroidal vasculitis. We speculate that PED may have been caused by choroidal hyperpermeability due to inflammation or choroidal vascular congestion.

Our SD-OCT images showed acutely changing retinal structures in the first few days after onset. Since treatment does not seem to affect the recovery of visual function in most patients, the influence of the therapy on the clinical course and the morphological changes of the retina may have been limited in our case. However, if time-course changes in OCT images of the retina in the acute stage are observed, the possibility of an influence of steroid treatment on the retinal structural changes should be considered.

Although high-resolution SD-OCT in addition to conventional diagnostics such as FA and indocyanine angiography have become available, diagnosis of APMPPE remains difficult. Evaluation of the clinical course was important for diagnosis in this case. Albeit, there is no clear etiology-based definition of APMPPE, it is a distinct clinical entity. The recent development of OCT has focused on detailed structures of the retina and choroid. Considering such technological advances, more detailed analyses of the ocular structure in APMPPE and other retinal inflammatory diseases are expected in the future.

\section{References}

1 Gass JDM: Acute posterior multifocal placoid pigment epitheliopathy. Arch Ophthalmol 1968;80:177-185.

$\longrightarrow 2$ Lee GE, Lee BW, Rao NA, Fawzi AA: Spectral domain optical coherence tomography and autofluorescence in a case of acute posterior multifocal placoid pigment epitheliopathy mimicking Vogt-Koyanagi-Harada disease: case report and review of literature. Ocul Immunol Inflamm 2011;19:42-47.

-3 Birnbaum AD, Blair MP, Tessler HH, et al: Subretinal fluid in acute posterior multifocal placoid pigment epitheliopathy. Retina 2010;30:810-814.

4 Gass JDM: Inflammatory disease of the retina and choroid; in Gass JDM (ed): Stereoscopic Atlas of Macular Disease: Diagnosis and Treatment. ed 3. St Louis, Mosby, 1987, pp 505-510.

5 Fiore T, Iaccheri B, Androudi S, et al: Acute posterior multifocal placoid pigment epitheliopathy. Retina 2009;29:994-1001. 
Tanigawa et al.: A Case of Acute Posterior Multifocal Placoid Pigment Epitheliopathy Demonstrating Vogt-Koyanagi-Harada Disease-Like Optical Coherence Tomography Findings in the Acute Stage

-6 Cheung CM, Yeo IY, Koh A: Photoreceptor changes in acute and resolved acute posterior multifocal placoid pigment epitheliopathy documented by spectral-domain optical coherence tomography. Arch Ophthalmol 2010;128:644-646.

$>7$ Sheufele TA, Witkin AJ, Schocket LS, et al: Photoreceptor atrophy in acute posterior multifocal placoid pigment epitheliopathy. Retina 2005;25:1109-1112.

-8 Garg S, Jampol LM: Macular serous detachment in acute posterior multifocal placoid pigment epitheliopathy. Retina 2004;24:650-651.

-9 Montero JA, Ruiz-Moreno JM, Fernandez-Munoz M: Spectral domain optical coherence tomography findings in acute posterior multifocal placoid pigment epitheliopathy. Ocul Immunol Inflamm 2011;19:48-50.

10 Lim LL, Watzke RC, Lauer AK, Smith JR: Ocular coherence tomography in acute posterior multifocal placoid pigment epitheliopathy. Clin Experiment Ophthalmol 2006;34:810-812.

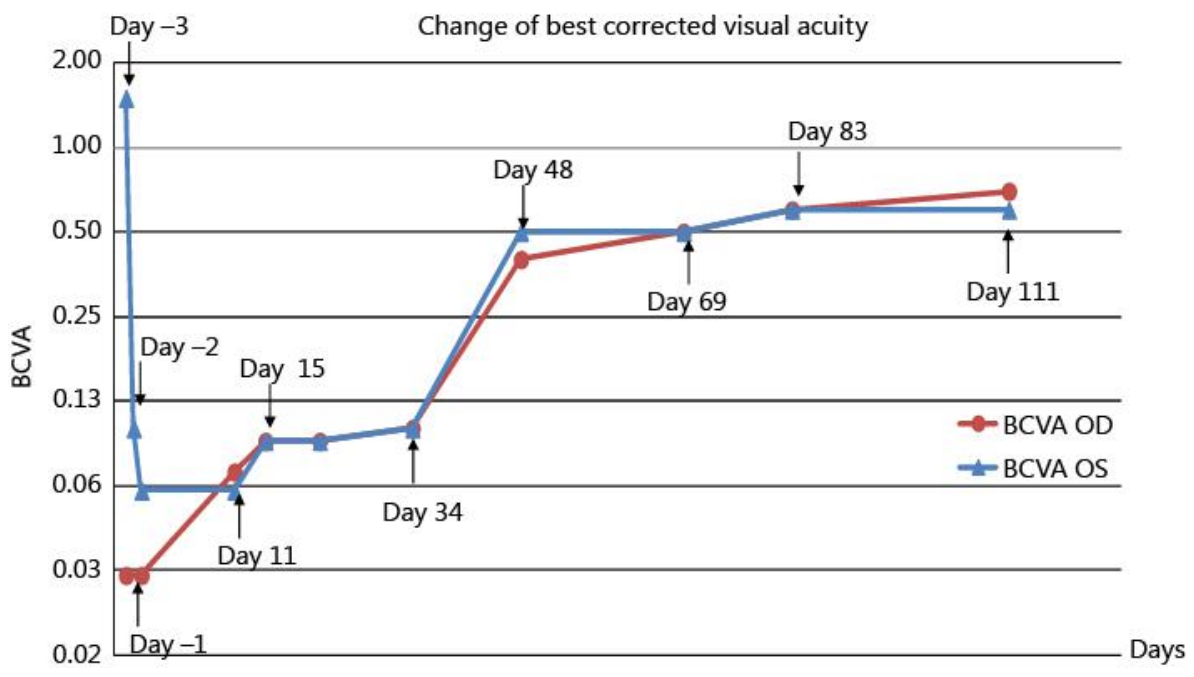

Fig. 1. Changes of BCVA throughout the disease course. Day -3 was the day the patient first visited our hospital, and it indicates the third day before the initiation of systemic steroid therapy. Day 0 indicates the first day of systemic steroid therapy. Day 11 indicates the 11th day after initiation of systemic steroid therapy. BCVA OD and BCVA OS indicate BCVA of the right and left eye, respectively. 
Case Reports in

Ophthalmology
Case Rep Ophthalmol 2013;4:172-179

DOI: $10.1159 / 000356051$

Tanigawa et al.: A Case of Acute Posterior Multifocal Placoid Pigment Epitheliopathy Demonstrating Vogt-Koyanagi-Harada Disease-Like Optical Coherence Tomography Findings in the Acute Stage
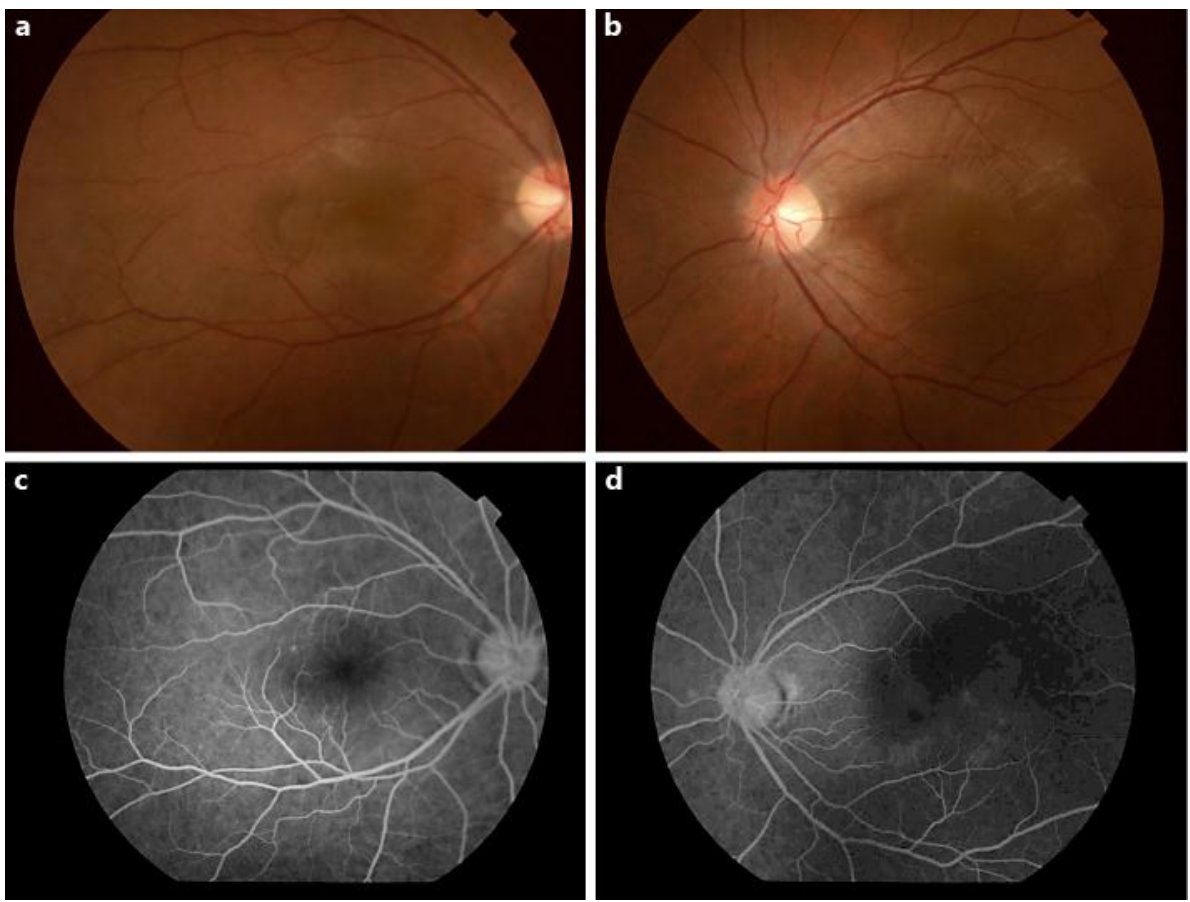

Fig. 2. Fundus photographs taken on day -1 . Day -1 indicates the day before the initiation of systemic steroid therapy. a, b Fundoscopy revealing yellow-white lesions in the posterior pole of both eyes. The lesions are mainly located in the macular area bilaterally. c, d Apparent hypofluorescence is unremarkable in the early phase of FA. 


\section{Case Reports in Ophthalmology}

\begin{tabular}{l|l}
\hline Case Rep Ophthalmol 2013;4:172-179 \\
\hline DOI: 10.1159/000356051 & $\begin{array}{l}\text { @ 2013 S. Karger AG, Basel } \\
\text { www.karger.com/cop }\end{array}$ \\
\hline
\end{tabular}

Tanigawa et al.: A Case of Acute Posterior Multifocal Placoid Pigment Epitheliopathy Demonstrating Vogt-Koyanagi-Harada Disease-Like Optical Coherence Tomography Findings in the Acute Stage
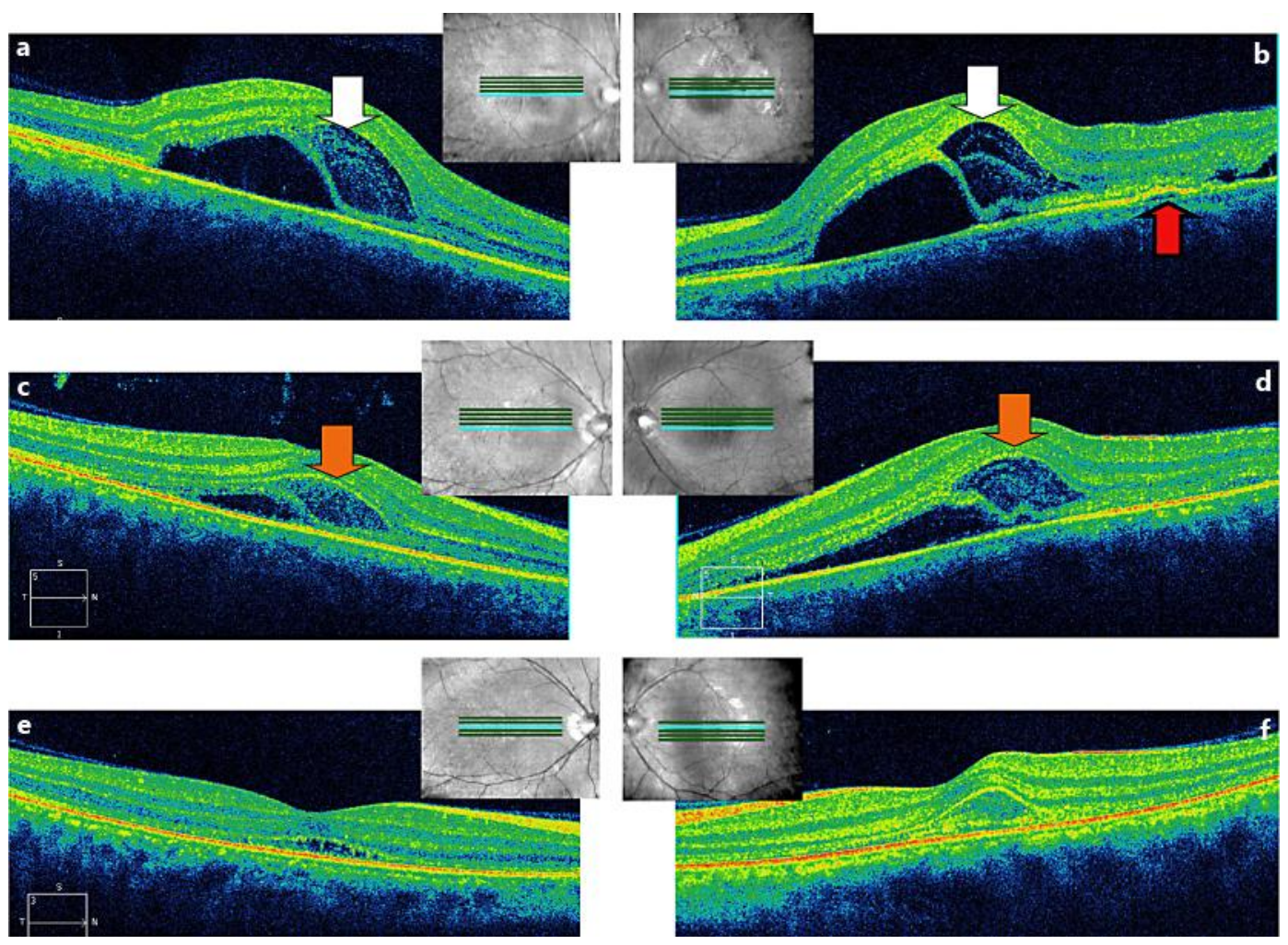

Fig. 3. a, b SD-OCT images on day -1. Day -1 indicates the day before the initiation of systemic steroid therapy. SD-OCT images of both eyes showing serous retinal detachment with a subretinal septum and an intraretinal compartmentalized cystic space. The compartmentalized intraretinal space was observed to contain two hyperreflective membranous layers (white arrow). In the left eye, PED was observed (red arrow). c, d On day 1, SD-OCT revealed that the subretinal fluid began to decrease. Disruption of the hyperreflective membranous layers in the intraretinal spaces (orange arrow) was observed. Day 1 indicates the first day after initiation of systemic steroid therapy. e, $\mathbf{f}$ On day 3 , the amount of subretinal fluid almost disappeared, but the IS/OS line remained disorganized in the affected area. Day 3 indicates the third day after initiation of systemic steroid therapy. 


\section{Case Reports in Ophthalmology}

\begin{tabular}{l|l}
\hline Case Rep Ophthalmol 2013;4:172-179 \\
\hline DOI: 10.1159/000356051 & $\begin{array}{l}\text { @ 2013 S. Karger AG, Basel } \\
\text { www.karger.com/cop }\end{array}$ \\
\hline
\end{tabular}

Tanigawa et al.: A Case of Acute Posterior Multifocal Placoid Pigment Epitheliopathy Demonstrating Vogt-Koyanagi-Harada Disease-Like Optical Coherence Tomography Findings in the Acute Stage
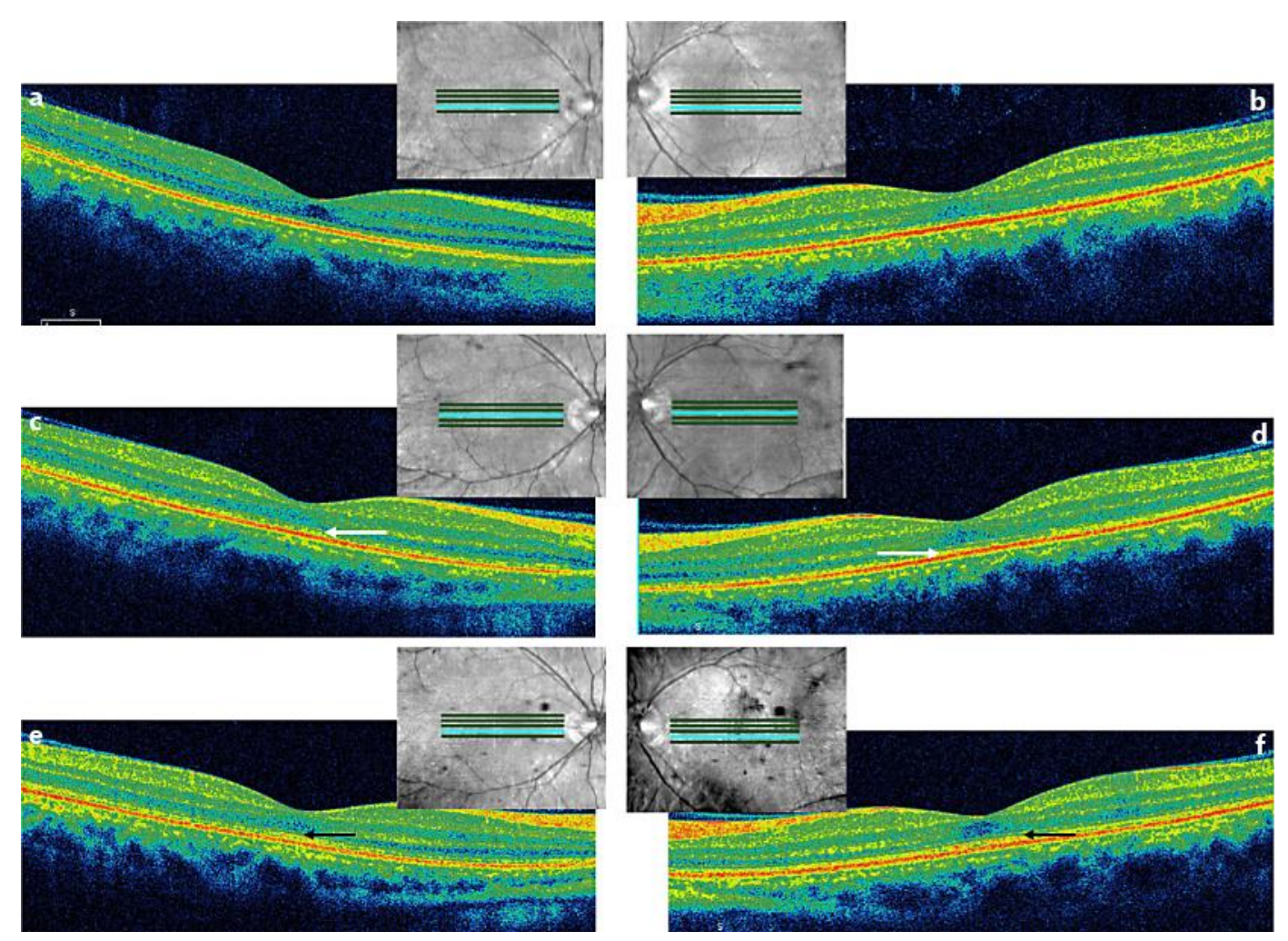

Fig. 4. a, b On day 15, OCT images showed that the IS/OS line remained disorganized, the retina appeared to be thin, and the outer nuclear layer appeared to be poorly reorganized. Day 15 indicates the 15 th day after initiation of systemic steroid therapy. c, d OCT detected partial recovery of the IS/OS line in the macular area of both eyes on day 48 (white arrow). Day 48 indicates the 48th day after the initiation of systemic steroid therapy. e, f Reorganization of the IS/OS line was observed by OCT on day 111 (black arrow). Day 111 indicates the 111st day after initiation of systemic steroid therapy. 